\title{
Medicine and Mathematics
}

\section{Statistics and ethics in medical research}

\author{
VI-Presentation of results
}

\author{
DOUGLAS G ALTMAN
}

A very important aspect of statistical method is the clear numerical and graphical presentation of results. Although many statistical textbooks and courses discuss simple visual methods such as histograms, bar charts, pie charts, and so on, they are usually introduced as descriptive or investigative techniques. It is uncommon to find discussion of how best to present the results of statistical analyses. This is surprising, since the interpretation of the results, both by the researcher and by later readers of the paper, may be critically dependent on the methods used to present the results.

Little need be said here about the simple visual methods already mentioned-they are well covered by Huff. ${ }^{1}$ The problems associated with graphs, however, are rather more important.

\section{Graphical presentation}

In 1976 a Government publication ${ }^{2}$ gave examples of some past successes in preventive medicine. One of these examples concerned the introduction in the 1930s of mass immunisation against diphtheria. Figure 1(a) shows their presentation of childhood mortality from diphtheria from 1871 to 1971 . This appears to show that the introduction of immunisation resulted in a rapid decline in mortality. In their figure, however, mortality is plotted on a logarithmic scale and shows proportional changes. When the data are plotted on a linear scale, ${ }^{3}$ as in fig $1(b)$, the visual effect is quite different, as is the interpretation. From this figure we can see that over the period in question mortality from diphtheria had been dropping very quickly, and this specific preventive measure was adopted relatively late in the day. This is not to say that the introduction of immunisation was not effective, but that the degree of its effectiveness that one accepts depends considerably on which way the data are presented.

For experimental data it is unlikely to be appropriate to transform the scale of one or both axes unless it has been necessary to carry out the analysis on transformed data. For example, if analysis has been carried out on log data, it is probably better to show a scatter diagram with a log scale to demonstrate that the transformed data comply with the appropriate assumptions.

\footnotetext{
Division of Computing and Statistics, Clinical Research Centre, Harrow, Middx HA1 3UJ

DOUGLAS G ALTMAN, BSC, medical statistician (member of scientific staff)
}

\section{Scatter diagrams and regression}

For simple data sets scatter diagrams are tremendously helpful. By showing all the data it is much easier for the reader to evaluate the analyses that were carried out. It is essential, however, that coincident points are indicated in some way. If there are different subgroups within the data set (different sexes perhaps) these may be indicated by means of different symbols. This will provide extra information at no expense, and will help to show the appropriateness (or otherwise) of analysing the data as one set, or for each subgroup separately.
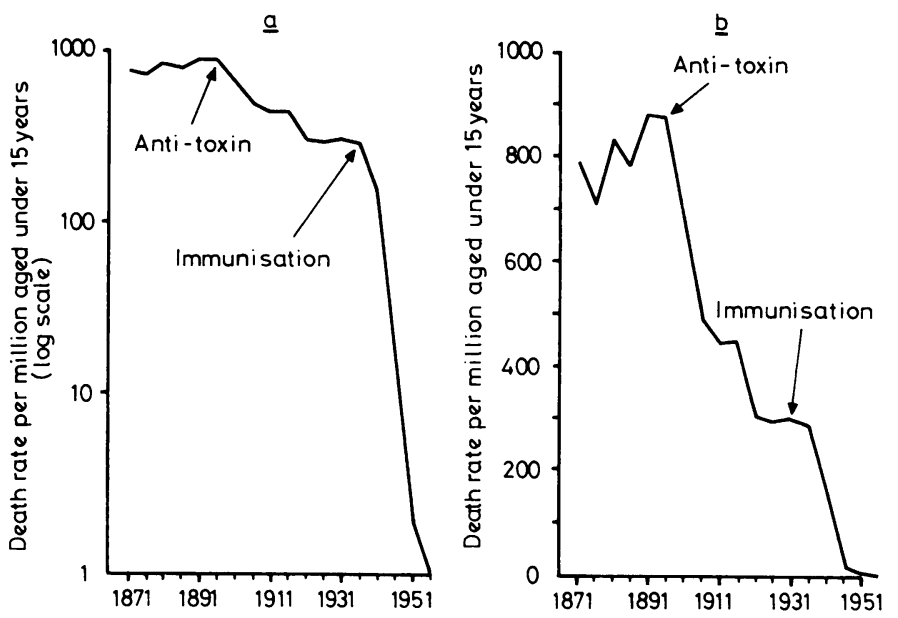

FIG 1 -Childhood mortality from diphtheria (a) on a $\log s^{2} \mathrm{lal}^{2}(\mathrm{~b})$ on a linear scale. ${ }^{3}$

Unfortunately, to many people scatter diagrams automatically suggest the calculation of correlations and the fitting of regression lines, even though one or both of these methods may be invalid or of no interest. One often sees scatter diagrams where a straight line has been drawn through the data but no reference is made to it, either in the figure or in the text. Perhaps the intention is to show that the data have been "properly analysed," but presentations like this demonstrate the reverse.

How should results of regression analyses be presented ? This will depend partly on the context. For example, if the analysis shows that the relationship between two variables is too weak to be of practical value, then there may be little point in quoting the equation of the line of best fit. If the equation is given then the standard error of the slope (and of the intercept if this is of practical importance) and the number of observations are important information. One other quantity is necessary, how- 
ever, before one can make full use of a regression equation. The equation can be used to estimate the variable $\mathrm{Y}$ for any new value of the variable $\mathrm{X}$. Such an estimate is, however, of limited value without some measure of its uncertainty, for which it is additionally necessary to have the residual standard deviation. ${ }^{4}$ This is a useful quantity in its own right, as it is a measure of the variability of the discrepancies (residuals) between the observations and the values predicted by the equation and is thus a measure of the "goodness of fit" of the regression line to the data. The residual standard deviation is rarely supplied in papers, so that it is impossible to know what uncertainty is attached to the use of the regression line for estimating $\mathrm{Y}$ from $\mathrm{X}$.

Whatever information is presented, it is vital that it is unambiguous. The following equation may be meant to give much of the information but the meaning of the last term is unclear:

$$
\operatorname{TBN}(\mathrm{g})=(28 \cdot 8 * \mathrm{FFM}(\mathrm{kg})+288) \pm 8 \cdot 5 \% \text {. }
$$

The paper ${ }^{5}$ from which this example comes also includes an example of a type of incorrect visual presentation of a regression equation-namely, the extension of the line well beyond the range of the data. This practice is extremely unreliable and potentially misleading, and can rarely be justified.

\section{Variability}

Despite its obvious importance and its almost universal presence in scientific papers, the presentation of variability in medical journals is a shambles. It is quite clear that some practices are now considered obligatory purely because they are widely used and accepted, not because they are particularly informative.

Much of the confusion may arise from imperfect appreciation of the difference between the standard deviation and the standard error. In simple terms the standard deviation is a measure of the variability of a set of observations, whereas the standard error is a measure of the precision of an estimate (mean, mean difference, regression slope, etc) in relation to its unknown true value. Despite this clear distinction in meaning, many people seem to have an innate preference for one or the other; some time ago I looked at all the issues of the $B M F$, Lancet, and New England Fournal of Medicine for October 1977 and found only three papers that used both, although 50 used either one or the other. Similar results were found in a much larger study. ${ }^{6}$ It has been suggested that perhaps the standard error of the mean is more popular because it is always much smaller, ${ }^{6}$ and this may well be so.

\section{STANDARD DEVIATION}

The standard deviation, which describes the variability of raw data, is often presented by attaching it to the corresponding mean using a \pm sign: "The mean ... was $30 \mathrm{mg}(S D \pm 4 \cdot 6$ $\mathrm{mg}$ )," or something similar. This presentation suggests that the standard deviation is $\pm 4.6 \mathrm{mg}$, but the standard deviation is always a positive number. ${ }^{8}$ More importantly, it also suggests that the range from mean $-S D$ to mean $+S D(25.4$ to $34.6 \mathrm{mg})$ is meaningful, but this is not so unless one is genuinely interested in the range encompassing about $68 \%$ of the observations. In general, the most useful range is probably the mean $\pm 2 \mathrm{SD}$, within which about $95 \%$ of the observations lie. This range is 20.8 to 39.2 , which is twice as wide as that implied by " \pm 4.6 mg." Such ranges apply only if the observations are approximately Normally distributed. Otherwise, although the standard deviation can be calculated, it may not convey much information about the spread of the data. In such cases the median and two centiles (say the 10th and 90th or the 5th and 95th for larger samples) will provide better information. ${ }^{910}$ The range of values may also be of interest, but it is highly dependent on the number of observations and is very sensitive to extreme or outlying observations. Alternatively, the omission of the \pm sign leads to an unambiguous although much less informative presentation: "The mean was $30 \mathrm{mg}$ (SD $4.6 \mathrm{mg}$ )."

\section{STANDARD ERRORS}

Similar comments apply to the presentation of standard errors. Here the most often quoted range of $\pm S E$ around an estimate is that within which we can be about $68 \%$ sure that the true value lies, whereas the $95 \%$ range is twice as wide. (For practical purposes these "confidence intervals" apply even when the data are not Normally distributed.) The presentation most usually used (mean $\pm S E$ ) is thus misleading in giving the impression of greater precision than has been achieved. Quoting the range mean $\pm 2 \mathrm{SE}$ is much better, but this is rarely seen. Much confusion would be eliminated if the sign \pm was used only when referring to a range.

\section{ERROR BARS}

Error bars are a popular way of displaying means and standard errors. They are usually a visual representation of the range mean $\pm \mathrm{SE}$ such as in fig 2 . In this example the error bars for $A$ and $B$ do not overlap: does this tell us anything about the difference between the groups?

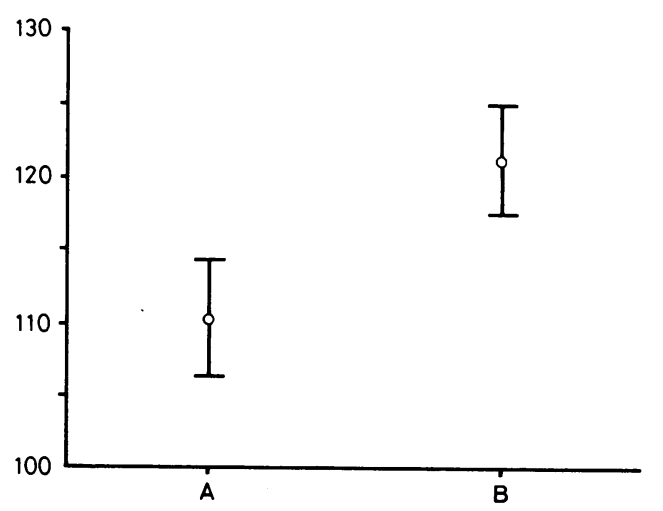

FIG 2-Mean ( \pm SE) diastolic blood pressure from two sets of observations.

Suppose A and B represent two different types of sphygmomanometer, and we measure the diastolic pressure of 15 people using each machine. Figure 3(a) shows the results of such an experiment where the agreement is clearly good, but machine $\mathrm{B}$ tends to give slightly higher readings. Figure $3(\mathrm{~b})$ shows some data where agreement is generally very poor. Yet both of these
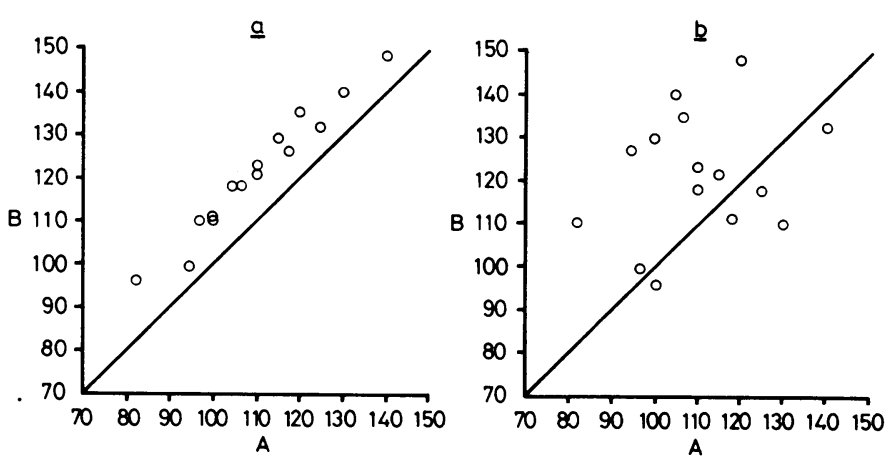

FIG 3-Comparison of diastolic blood pressures measured by two sphygmomanometers on 15 subjects (a) with good agreement but some bias (b) with very poor agreement. 
sets of data can be described exactly by the means and SEs in fig 2. This is because fig 2 tells us nothing about differences between machines for each subject. Error bars are thus useless in the case of paired observations.

Now suppose that we wish to compare the diastolic blood pressures of two distinct groups of people, say doctors (group A) and bus-drivers (group B). Figures 4(a) and 4(b) show two possible outcomes. In which case, if either, are the two groups significantly different ? It is not easy to tell from the raw data shown that the groups are significantly different in fig 4(a) $(p<0.05)$ but not in fig 4(b) $(p>0.1)$. What would an "errorbar" plot show? Well, again both examples would yield fig 2 , showing that the visual impression of non-overlapping bars does not by itself give any information about statistical significance. If the error bars do overlap, however, then the difference between the means is not statistically significant. ${ }^{11}$

For error bars to be useful they ought to convey useful information about either the precision of individual means or the differences between means. In their usual form they do neither, although my impression is that many people believe that they do both. The use of confidence intervals (mean $\pm 2 \mathrm{SE}$ ) instead of error bars does at least give useful information about individual means. Although it is sometimes possible to make the visual presentation give an indication of statistical significance, it is probably better to give confidence intervals and, if desired, report on the significance separately.
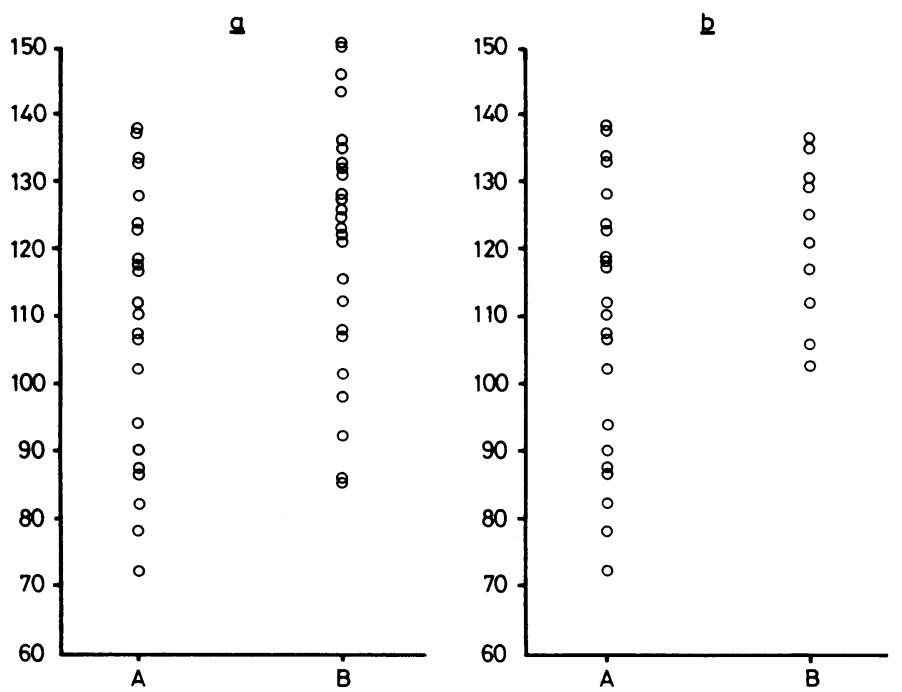

FIG 4 (a) and (b)-Comparisons of diastolic blood pressure in two different groups of subjects.

\section{Numerical precision}

One other aspect of presentation that deserves some comment is numerical precision. It is rarely necessary to quote resultsmeans, standard deviations, and so on-to more than three significant figures (that is, excluding leading or trailing zeros). For tabular presentation it may be a positive advantage to reduce the precision of each entry to make any patterns or trends more obvious. ${ }^{12}$

Spurious precision should also be avoided. Examples are the quoting of $t$ or $\chi^{2}$ values to four decimal places, and a regression slope with seven significant figures (12.97642). My favourite is the summary ${ }^{18}$ of a test of significance as $\mathrm{p}<10^{-54}$, although I must concede that there is only one significant figure here!

\section{Some suggestions}

More thought should be given to numerical and visual presentation, rather than automatically following precedent.
Some ways of supplying more information without using more space are:

(1) In a plot information about the spread of data (by \pm 2 SD ranges or centiles) can be given as well as means and confidence intervals.

(2) A figure and a table may be combined by using the $\mathrm{X}$ axis labels as table column headings. For example, in fig 2 I could have given the mean, $\mathrm{SD}$, range, and sample size for the two groups under the figure using little extra space.

(3) When scatter plots have the same variable on each axis as in fig 3(a) and 3(b), a small histogram of the within-person differences can be added in an otherwise empty corner.

\section{Summary}

Whatever results are presented it is vital that the methods are identified. In one survey of over 1000 papers $^{14}$ as many as $20 \%$ of the procedures were unidentified, and in another it was not clear whether the SD or SE was given in $11 \%$ of 608 papers. ${ }^{6}$ It is impossible to appraise a paper in the presence of such ambiguities.

Visual display is a particularly effective way of presenting results. Given alternatives, however, many people might opt for the method of display that fits in better with their beliefs. If decisions are taken as a result of such presentations then there is scope for manipulating events by choice of presentation. This practice is well recognised in the way statistics are sometimes presented in the mass media and advertisements; we should not rule out this phenomenon in the medical world.

This is the sixth in a series of eight articles. No reprints will be available from the authors.

\section{References}

1 Huff D. How to lie with statistics. Harmondsworth: Penguin, 1973.

Department of Health and Social Security. Prevention and health: everybody's business. London: HMSO, 1976.

${ }^{3}$ Radical Statistics Health Group. Whose priorities? London: Radical Statistics, 1976.

- Armitage P. Statistics in medical research. Oxford: Blackwell, 1971:150-6.

5 Hill GL, Bradley JA, Collins JP, McCarthy I, Oxby CB, Burkinshaw L. Fat-free body mass from skinfold thickness: a close relationship with total body nitrogen. Br $\mathcal{F}$ Nutr 1978;39:403-5.

6 Bunce H, Hokanson JA, Weiss GB. Avoiding ambiguity when reporting variability in biomedical data. Am $\mathcal{F}$ Med 1980;69:8-9.

7 Glantz SA. Biostatistics: how to detect, correct and prevent errors in the medical literature. Circulation 1980;61:1-7.

${ }^{8}$ Gardner MJ. Understanding and presenting variation. Lancet 1975 ; : $: 230-1$.

9 Mainland D. SI units and acidity. $B r$ Med f 1977; ;ii:1219-20.

10 Feinstein AR Clinical biostatistics. XXXVII Demeaned errors, confidence games, nonplussed minuses, inefficient coefficients, and other statistical disruptions of scientific communication. Clin Pharmacol Ther $1976 ; 20: 617-31$.

11 Browne RH. On visual assessment of the significance of a mean difference. Biometrics $1979 ; 35: 657-65$.

12 Ehrenberg ASC. Rudiments of numeracy. Fournal of the Royal Statistical Society Series A 1977;140:277-97.

13 Vaughan Williams EM, Tasgal J, Raine AEG. Morphometric changes in rabbit ventricular myocardium produced by long-term beta-adrenoceptor blockade. Lancet 1977 ;ii:850-2.

${ }^{14}$ Feinstein AR. Clinical biostatistics. XXV A survey of the statistical procedures in general medical journals. Clin Pharmacol Ther 1974;15: 97-107.

\section{Correction}

\section{Evaluation of a patient education manual}

The authors of this paper (4 October, p 924) wish to apologise for inadvertently failing to acknowledge the important contribution of Dr Mick Murray in constructing the questionnaires used in the study and his advice on the study design. 